\title{
Expressions of fatty acid synthase and HER2 are correlated with poor prognosis of ovarian cancer
}

\author{
Yunlang Cai $\cdot$ Jingmei Wang $\cdot$ Lin Zhang $\cdot$ Di Wu $\cdot$ \\ Dandan Yu $\cdot$ Xiaoqiang Tian $\cdot$ Jun Liu $\cdot$ Xinru Jiang $\cdot$ \\ Yang Shen $\cdot$ Lihua Zhang $\cdot$ Mulan Ren $\cdot$ Peilin Huang
}

Received: 15 November 2014 / Accepted: 19 November 2014/Published online: 30 November 2014

(C) The Author(s) 2014. This article is published with open access at Springerlink.com

\begin{abstract}
The present study was designed to explore the cross talk between fatty acid synthase (FASN) and HER2 (ErbB2) in ovarian cancer. A total of 60 ovarian cancer patients and 15 normal ovarian tissues were enrolled. Tissue array was conducted by using a tissue microarray instrument. Immunohistochemistry was performed to quantify the expressions of HER2 and FASN. The FASN was detected to be distributed in the cell cytoplasm and was significantly correlated with cancer grade $(p=0.000)$ and FIGO staging $(p=0.000)$. Patients with FASN overexpression in ovarian cancer tend to have a worse overall survival rate $(p=0.000)$. HER2 was also stained to be distributed in the cell cytoplasm associated with higher expression in high-grade cancer. It was also disclosed that FASN expression level is not correlated with HER2 status
\end{abstract}

Y. Cai $\cdot$ L. Zhang $\cdot$ Y. Shen $\cdot$ M. Ren

Department of Obestetrics and Gynecology, Zhongda Hospital, Southeast University, Dingjia Qiao Road 87,

Nanjing 210009, Jiangsu Province, China

J. Wang

Department of Pathology, Drum Tower Hospital,

Nanjing 210009, Jiangsu Province, China

D. Wu $\cdot$ D. Yu $\cdot$ J. Liu $\cdot$ X. Jiang $\cdot$ P. Huang $(\bowtie)$

Medical College, Southeast University, Dingjia Qiao Road 87,

Nanjing 210009, Jiangsu Province, China

e-mail: peilinhuangnanjing@yeah.net

X. Tian

The Second Hospital of Nanjing, Zhongfu Road 1,

Nanjing 210003, Jiangsu Province, China

L. Zhang

Department of Pathology, Zhongda Hospital, Southeast

University, Dingjia Qiao Road 87,

Nanjing 210009, Jiangsu Province, China in ovarian cancer. These results for the first time indicated that a cross talk in FASN and HER2 expressions might be associated with prognosis in malignant ovarian cancer.

Keywords Fatty acid synthase - HER2 - Ovarian cancer · Clinicopathological factors

\section{Introduction}

Fatty acid synthase (FASN), one of the most important biosynthetic enzymes in lipogenesis, is regulated by hormones, growth factors and diet. Little or no FASN expression is disclosed to be in normal cells and tissues except for liver and adipose tissue [1]. However, FASN expression is often upregulated in rapid proliferation cells. Inhibition of FASN expression could repress cell proliferation in various cancers [2]. For those reasons, FASN has become as an attractive target for cancer therapy in last 15 years [3].

HER2 (ErbB2) is a member of the epidermal growth factor receptor (EGFR) family of receptor tyrosine kinases (RTKs) that play a pivotal role in oncobiological processes [4]. The "HER2-PI3K/Akt-FASN axis"' is involved in the regulation of malignant phenotype in colorectal cancer cells [5]. It is reported that a cross talk between ErbB and FASN mediates ovarian cancer cells proliferation [6]. Mounting evidences indicate that FASN gene network and HER2 oncogene system have synergetic effect in tumorigenesis. We speculated that whether an ErbB/FASN cross talk plays a vital role in mediating malignant phenotype of ovarian cancer. Tissue array and immunohistochemistry were used for analysis of FASN and HER2 expressions, and the correlations of FASN and HER2 expressions with clinicopathological factors were statistically analyzed. 


\section{Materials and methods}

Patients

This study was approved by the Ethics Committee of ZhongDa Hospital, and the informed consent was obtained from all subjects. All experiments were performed in accordance with relevant guidelines and regulations. Patients were treated in the ZhongDa Hospital between 1997 and 2000. The study population is consisted of 60 ovarian cancer patients; 15 normal ovarian tissues were used for control group. The summary of patient's characteristics is shown in (Table 1).

Tissue array

Tumor tissues were obtained at the first laparotomy with no neoadjuvant chemotherapy and any other treatment. Slides stained with hematoxylin and eosin $(\mathrm{H}-\mathrm{E})$ were generated from the original paraffin blocks that were analyzed for the diagnosis of epithelial ovarian cancer (EOC). Tissue microarray (TMA) was designed after selection of the most representative areas by pathologist. For each block, triplicate $0.8 \mathrm{~mm}$ cores of tumor were placed on a TMA, which was performed by Dr. Wangjinei (Drum Tower Hospital, Nanjing, China) using a TMA instrument.

Immunohistochemistry

The tissue array was hydrated in gradient alcohol, and antigen retrieval was performed in ethylenediaminetetraacetic acid (EDTA)-containing antigen retrieval buffer $(\mathrm{pH}=8.0)$ in $95{ }^{\circ} \mathrm{C}$ followed by $3 \% \mathrm{H}_{2} \mathrm{O}_{2}$ incubation for $30 \mathrm{~min}$. After blocking by goat serum for $10 \mathrm{~min}$, mouse anti-human FASN monoclonal antibody and mouse antihuman HER2 monoclonal antibody were incubated overnight at $4{ }^{\circ} \mathrm{C}$. The corresponding Horseradish peroxidase (HRP)-conjugated secondary antibodies were incubated for $30 \mathrm{~min}$ at room temperature before visualization by diaminobenzidine (DAB) reagent. The tissue array slides then were stained with hematoxylin and mounted with slide cover for microscopic evaluation. FASN and HER2 staining intensity were scored independently by two observers. Immunoreactivity was scored as follows: 0 (undetectable), + (weakly positive), ++ (moderately positive), +++ (intensely positive).
Table 1 Association between FASN and HER2 expressions and clinicopathological factors in patients with ovarian clear cell carcinoma

\begin{tabular}{|c|c|c|c|c|c|c|c|}
\hline \multirow[t]{2}{*}{ Factors } & \multirow[t]{2}{*}{ Patients } & \multicolumn{3}{|c|}{ FASN } & \multicolumn{3}{|c|}{ HER2 } \\
\hline & & High & Low & & High & Low & \\
\hline \multicolumn{8}{|l|}{ Histological type } \\
\hline Serous & 64 & 49 & 15 & $p=0.06$ & 20 & 44 & $p=0.372$ \\
\hline Mucinous & 14 & 10 & 4 & & 7 & 7 & \\
\hline Endometrioid & 17 & 8 & 9 & & 5 & 12 & \\
\hline \multicolumn{8}{|l|}{ Cancer grade } \\
\hline G1 & 21 & 10 & 16 & $p=0.000$ & 8 & 13 & $p=0.887$ \\
\hline $\mathrm{G} 2$ & 12 & 5 & 7 & & 4 & 8 & \\
\hline G3 & 62 & 52 & 10 & & 20 & 42 & \\
\hline \multicolumn{8}{|l|}{ FIGO stage } \\
\hline I & 8 & 1 & 7 & $p=0.000$ & 1 & 7 & $p=0.204$ \\
\hline II & 10 & 3 & 7 & & 3 & 7 & \\
\hline III & 60 & 45 & 15 & & 19 & 41 & \\
\hline IV & 17 & 16 & 1 & & 9 & 8 & \\
\hline \multicolumn{8}{|l|}{ Residual tumor } \\
\hline$<1 \mathrm{~cm}$ & 68 & & & & & & \\
\hline$\geq 1 \mathrm{~cm}$ & 27 & & & & & & \\
\hline \multicolumn{8}{|l|}{ Age (years) } \\
\hline$<50$ & 38 & & & & & & \\
\hline$\geq 50$ & 57 & & & & & & \\
\hline \multicolumn{8}{|l|}{ HER2 } \\
\hline High & 32 & & & & & & \\
\hline Low & 63 & & & & & & \\
\hline
\end{tabular}


Statistical analyses

The data were statistically analyzed with SPSS 16.0 software. The correlation analysis was performed with Spearman's test. $p<0.05$ was considered as statistically significant.

\section{Results}

Relationship between FASN expression and clinicopathological factor in ovarian cancer

FASN was detected to be distributed in the cell cytoplasm and high FASN expression levels were observed in $70.5 \%$ (67/95) of analyzed tumors. There was no FASN immunoreactivity (0/15) in the normal ovarian tissues (Fig. 1). Depending on FASN immunostaining score, patients were divided into high expression $(2+$ and $3+$ ) and low expression $(-$ and + ) groups. There was no significant association between FASN status and histological type $(p=0.06)$, patients age $(p=0.650)$, residual tumor $(p=0.455)$. However, cancer grade $(p=0.000)$ and FIGO staging $(p=0.000)$ were significantly correlated with FASN expression (Table 1). Patients with FASN overexpression in ovarian cancer tend to have a worse overall survival rate $(p=0.000)$ (Fig. 2).

\section{Relationship between HER2 expression}

and clinicopathological factor in ovarian cancer

In the pathological section, HER2 was also stained to be distributed in the cell cytoplasm (Fig. 1). Only one normal ovarian tissue shows HER2 positive results $(+)(1 / 15)$. There was no significant association between HER2 status and patient age $(p=0.826)$, residual tumor $(p=0.471)$, histological type $(p=0.372)$, cancer grade $(p=0.887)$ and FIGO staging $(p=0.077)$. HER2 has more intensity expression in high-grade cancer (Table 1). However, no statistically significance with respect to all tumors $(p=0.077)$ (Fig. 3).

FASN expression level is not correlated with HER2 status in ovarian cancer

We analyzed the relationship between FASN and HER2 expressions. According to ICH scores in tissues array, there was no significant correlation in ovarian cancer $(p=0.385)$. Nevertheless, to confirm the finding that FASN and HER2 expressions were correlated in ovarian
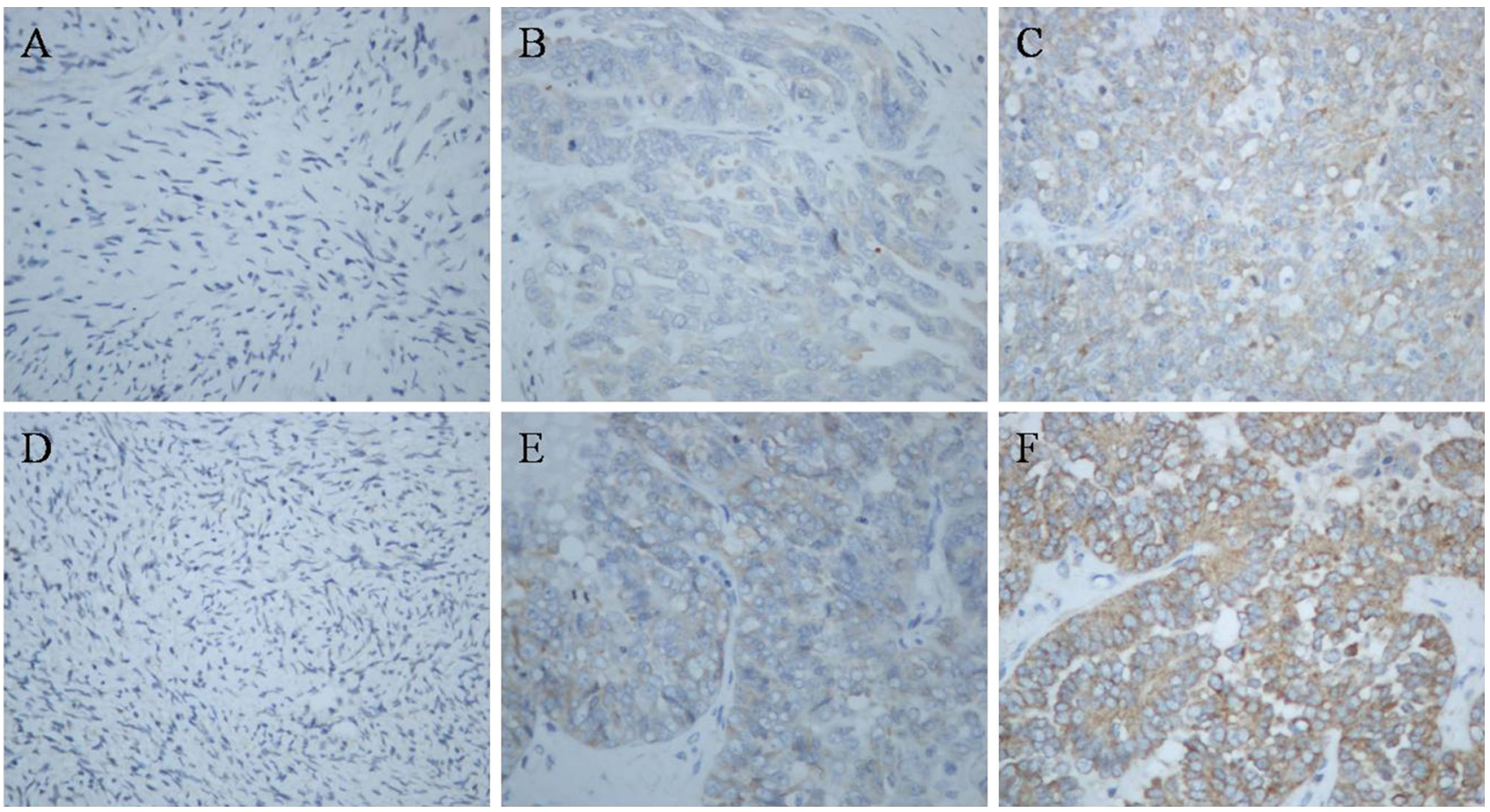

Fig. 1 Immunostaining showing regional diversity of FASN and HER2 expressions in ovarian tissues and ovarian cancers. Immunostaining showing regional diversity of FASN/HER2 expression in ovarian tissues $(\mathbf{a} / \mathbf{d})$. The expression levels were graded in a 4-point scale. 0 (undetectable), + (weakly positive), ++ (moderately positive), and +++ (intensely positive); Low $(0$ and +$)$ and high $(++$ and +++$)$ expressions of FASN in tissue samples $(\mathbf{b}, \mathbf{c})$. Low $(0$ and +$)$ and high $(++$ and +++$)$ expressions of HER2 in tissue samples $(\mathbf{e}, \mathbf{f})$ 


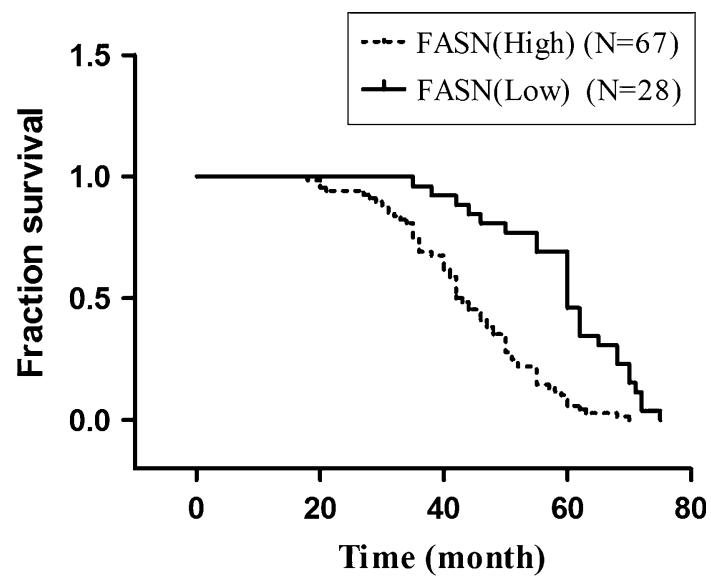

Fig. 2 Overall survival based on the expression of FASN. KaplanMeier survival analysis showing that high expression of FASN (++ and +++ ) was associated with a shorter overall survival in comparison with low FASN expression $(0$ and +$)$ in ovarian cancer $(p=0.000$, log-rank test $)$

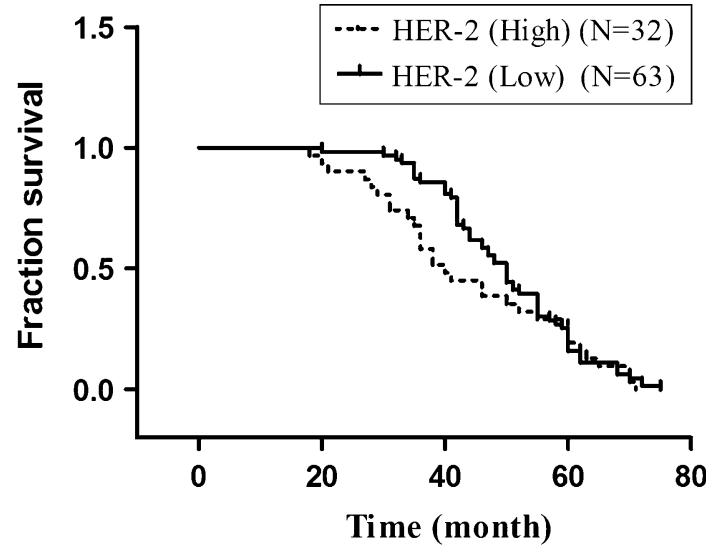

Fig. 3 Overall survival based on the expression of HER2. KaplanMeier survival analysis showing that high expression of HER2 $(++$ and +++ ) was not associated with overall survival in comparison with low HER 2 expression $(0$ and +$)$ in ovarian cancer $(p=0.272$, log-rank test)

cancer, patients were divided into high expression of FASH (high)/HER2 (high) and FASN (high)/HER2 (Low) group (Fig. 4). There was worse overall survival in FASH (high)/ HER2 (high) group $(p=0.002)$ (Fig. 5).

\section{Discussion}

Abnormal cell growth is one of the characteristics of cancer cells; the rapid growth of cells requires energy metabolism support [7]. The present study focuses on the correlation between ovarian cancer and expressions of FASN and HER2. FASN is the key enzyme involved in the regulation of cellular fatty acid synthesis and is highly expressed in

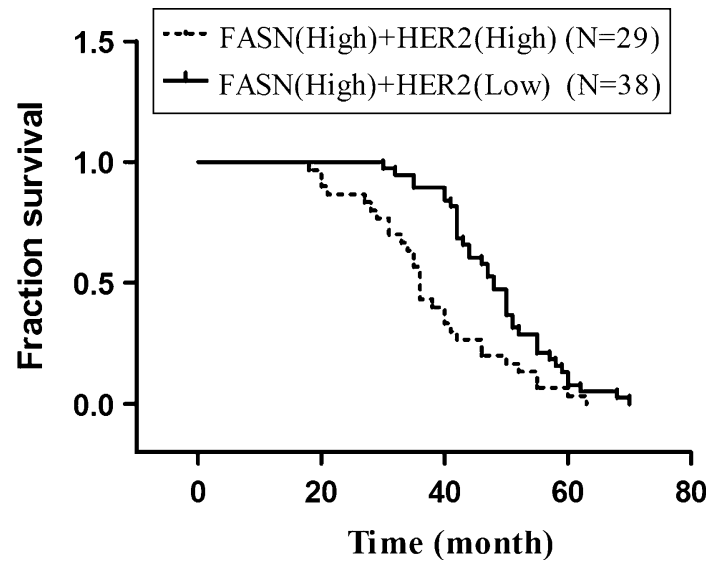

Fig. 4 Overall survival based on the expression of FASN and HER2. To confirm the finding that FASN and HER2 expressions were correlated in ovarian cancer, patients were divided into high expression of FASH (high)/HER2 (high) and FASN (high)/HER2 (low) group. Kaplan-Meier survival analysis showing that FASH (high)/HER2 (high) group was associated with a shorter overall survival in comparison with low FASN (high)/HER2 (low) group in ovarian cancer ( $p=0.002$, log-rank test)

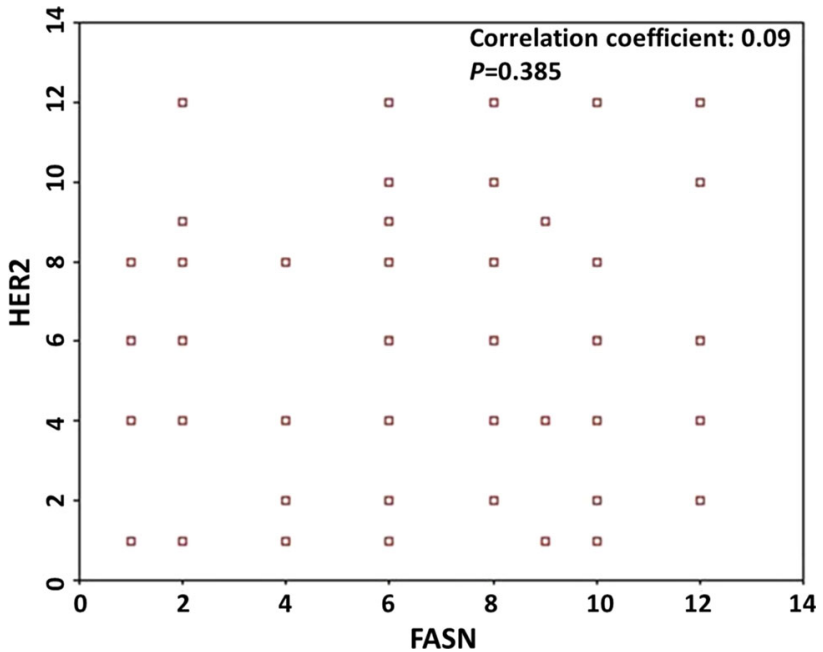

Fig. 5 Correlation of expression FASN and HER2 in ovarian cancer. To investigate the expression correlation of FASN and HER2 in ovarian cancer, Spearman correlation analysis showed that FASN and HER2 expressions have no correlation in ovarian cancer $(p=0.385$, CC: 0.09)

many cancers such as lung cancer, breast cancer, prostate cancer, colon cancer and many other tumors [8-11]. In the study, we found that FASN has abnormally high expression in ovarian cancer, which was consistent with previous report [12]. High stage of cancer cells often has faster proliferative capacity. A significantly increase in FASN expression in high stage of ovarian cancer was presented, which could be due to the fact that overexpression of FASN can provide material and energy for the rapid proliferation of tumor. Gansler TS deemed that FASN was an 
independent prognostic indicator for tumor [12]. The present results revealed that the intensity of FASN expression and survival rate of ovarian cancer patients were closely linked, although further study is necessitated to ascertain the critical role of FASN as a prognostic marker for ovarian cancer.

HER2 is a proto-oncogene that controls cell proliferation [4]; there is a large difference in the expression of HER2 in ovarian cancer, the overall scope of the present report is 5-30\% [13-16]. In this research, we outlined that the overall expression of HER2 in ovarian carcinoma was about $33 \%$. Most of the literature suggests HER2 protein overexpression is associated with poor prognosis in ovarian cancer with a single factor analysis. This was reflected that HER2 overexpression is correlated with increased risk of death, shortened disease progression-free survival and overall survival rate in patients $[17,18]$. Nevertheless, the results of multivariate analysis are still controversial. Camilleri-Broet believed that HER2 protein expression is the independent prognostic factors via multivariate analysis [19]. However, Riener EK reported that HER2 overexpression is not an independent prognostic factor for ovarian cancer, and its role for predicting disease needs to rest on other factors, such as, clinical stage, histological grade and residual tumor volume [16]. We found that there was positive rate increased in high stage of ovarian cancer. The patients with high expression of HER2 tended to acquire worse survival rate. The difference was found to be no statistically significant. This suggests that HER2 may be able to influence the prognosis of patients. These findings should be interpreted with caution. Resulting in the variety of HER2 expression and the differences may be associated with different affinity of antibody, clinical pathology laboratory, racial, tumor staging, evaluation standard of HER2 positive evaluation standard and sample. HER2 is likely to become an independent risk factor for prognosis; further evaluations are required in the near future.

Some literature for ovarian cancer suggested that FASN could regulate the expression of HER2 through PI3k-Akt pathway [20]. Similarly, HER2 can regulate the expression of FASN through the reverse pathway [21]. This suggested that FASN and HER2 might have a mutual role in triggering the tumor. The present study suggested no obvious correlation between FASN and HER2 expressions, and this might be due to the relatively small size of samples. However, we found simultaneously that the 5-year survival rate in patients with FASN and HER2 expressions was comparatively lower than that in patients with a single expression. Co-expression of FASN and HER2 might be a sign of poor prognosis tumor.

In the present study, we establish an association between the prognosis of patients with ovarian cancer and FASN/ HER2 expressions in ovarian tissue. FASN and HER2 expressions might be associated with prognosis in malignant ovarian cancer.

\section{Conclusions}

We firstly put the first evidence that a cross talk in FASN and HER2 expressions might be associated with prognosis in malignant ovarian cancer.

Acknowledgments This research was supported by the National Natural Science Foundation of China (30973475) and the Natural Science Foundation of JiangSu Province (BK2012749) and Maternal and Jiangsu Provincial Maternal and child health research project of JiangSu Health Department (F201351).

Conflict of interest The authors declare that they have no conflict of interests.

Open Access This article is distributed under the terms of the Creative Commons Attribution License which permits any use, distribution, and reproduction in any medium, provided the original author(s) and the source are credited.

\section{References}

1. Menendez JA, Lupu R. Fatty acid synthase and the lipogenic phenotype in cancer pathogenesis. Nat Rev Cancer. 2007;7:76377.

2. Li N, Bu X, Tian X, Wu P, Yang L, Huang P. Fatty acid synthase regulates proliferation and migration of colorectal cancer cells via HER2-PI3K/Akt signaling pathway. Nutr Cancer. 2012;64: 864-70.

3. Rahman MT, Nakayama K, Rahman M, Katagiri H, Katagiri A, Ishibashi $\mathrm{T}$, et al. Fatty acid synthase expression associated with NAC1 is a potential therapeutic target in ovarian clear cell carcinomas. Br J Cancer. 2012;107:300-7.

4. Hung MC, Lau YK. Basic science of HER-2/neu: a review. Semin Oncol. 1999;26:51-9.

5. Li N, Lu H, Chen C, Bu X, Huang P. Loss of fatty acid synthase inhibits the "HER2-PI3K/Akt axis" activity and malignant phenotype of Caco-2 cells. Lipids Health Dis. 2013;1:83.

6. Grunt TW, Wagner R, Grusch M, Berger W, Singer CF, Marian $B$, et al. Interaction between fatty acid synthase- and ErbB-systems in ovarian cancer cells. Biochem Biophys Res Commun. 2013;385:454-9.

7. Malthièry Y, Savagner F. Energy metabolism of the cancer cell: example of mitochondria-rich endocrine tumors. Ann Endocrinol (Paris). 2006;67:205-13.

8. Piyathilake CJ, Frost AR, Manne U, Bell WC, Weiss H, Heimburger DC, et al. The expression of fatty acid synthase (FASE) is an earlyevent in the development and progression of squamous cell carcinoma of the lung. Hum Pathol. 2000;31:1068-73.

9. Ogino S, Nosho K, Meyerhardt JA, Kirkner GJ, Chan AT, Kawasaki $\mathrm{T}$, et al. Cohort study of fatty acid synthase expression and patient survival in colon cancer. J Clin Oncol. 2008;26: 5713-20.

10. Rossi S, Graner E, Febbo P, Weinstein L, Bhattacharya N, Onody $\mathrm{T}$, et al. Fatty acid synthase expression defines distinct molecular signatures in prostate cancer. Mol Cancer Res. 2003;1:707-15.

11. Pizer ES, Thupari J, Han WF, Pinn ML, Chrest FJ, Frehywot GL, et al. Malonyl-coenzyme-A is a potential mediator of cytotoxicity 
induced by fatty-acid synthase inhibition in human breast cancer cells and xenografts. Cancer Res. 2000;60:213-8.

12. Gansler TS, Hardman W 3rd, Hunt DA, et al. Increased expression of fatty acid synthase (OA-519) in ovarian neoplasms predicts shorter survival. Hum Pathol. 1997;28:686-92.

13. Verri E, Guglielmini P, Puntoni M, Perdelli L, Papadia A, Lorenzi P, et al. HER2/neu oncoprotein overexpression in epithelial ovarian cancer: evaluation of its prevalence and prognostic significance. Clinical study. Oncology. 2005;68:154-61.

14. Tanabe H, Nishii H, Sakata A, Suzuki K, Mori Y, Shinozaki H, et al. Overexpression of HER-2/neu is not a risk factor in ovarian clear cell adenocarcinoma. Gynecol Oncol. 2005;94:735-9.

15. Mano MS, Awada A, Di Leo A, Durbecq V, Paesmans M, Cardoso F, et al. Rates of topoisomerase II-alpha and HER-2 gene amplification and expression in epithelial ovarian carcinoma. Gynecol Oncol. 2004;92:887-95.

16. Riener EK, Arnold N, Kommoss F, Lauinger S, Pfisterer J. The prognostic and predictive value of immunohistochemically detected HER-2/neu overexpression in 361 patients with ovarian cancer: a multicenter study. Gynecol Oncol. 2004;95:89-94.

17. Hogdall EV, Christensen L, Kjaer SK, Blaakaer J, Bock JE, Glud E, et al. Distribution of HER-2 overexpression in ovarian carcinoma tissue and its prognostic value in patients with ovarian carcinoma: from the Danish MALOVA Ovarian Cancer Study. Cancer. 2003;98:66-73.

18. Wang Y, Kristensen GB, Helland A, Nesland JM, Børresen-Dale AL, Holm R. Protein expression and prognostic value of genes in the erb-b signaling pathway in advanced ovarian carcinomas. Am J Clin Pathol. 2005;124:392-401.

19. Camilleri-Broet S, Hardy-Bessard AC, Le Tourneau A, Paraiso D, Levrel O, Leduc B, et al. HER-2 overexpression is an independent marker of poor prognosis of advanced primary ovarian carcinoma: a multicenter study of the GINECO group. Ann Oncol. 2004;15:104-12.

20. Vazquez-Martin A, Colomer R, Brunet J, Lupu R, Menendez JA. Overexpression of fatty acid synthase gene activates HER1/ HER 2 tyrosine kinase receptors in human breast epithelial cells. Cell Prolif. 2008;41:59-85.

21. Menendez JA, Vellon L, Mehmi I, Oza BP, Ropero S, Colomer $\mathrm{R}$, et al. Inhibition of fatty acid synthase (FAS) suppresses Her2/ neu (ErbB-2) oncogene overexpression in cancer cells. Proc Natl Acad Sci USA. 2004;101:10715-20. 